\title{
Advances in Immunotherapy of Malignant Pleural Mesothelioma
}

\author{
Dongying Liao ${ }^{1,2}$ \\ Yongchao $\mathrm{Yu}^{1,2}$ \\ Qingyun Mei ${ }^{1,2}$ \\ Ziwei Wang ${ }^{1,2}$ \\ Xiaojiang $\mathrm{Li}^{1,2}$ \\ Yingjie Jia ${ }^{1,2}$ \\ Fanming Kong ${ }^{1,2}$
}

'Department of Oncology, First Teaching Hospital of Tianjin University of Traditional Chinese Medicine, Nankai District, Tianjin, 300193, People's Republic of China; ${ }^{2}$ National Clinical Research Center for Chinese Medicine Acupuncture and Moxibustion, Nankai District, Tianjin, 300193, People's Republic of China
Correspondence: Fanming Kong

Department of Oncology, First Teaching Hospital of Tianjin University of

Traditional Chinese Medicine, Anshanxi

Road, Nankai District, Tianjin, 300193,

People's Republic of China

Tel + 8613652123653

Email kongfanming08@I63.com

\begin{abstract}
Malignant pleural mesothelioma (MPM) represents the uncommon cancer originating from pleural mesothelial cells, which is associated with dismal prognostic outcome. According to CheckMate-743 results, nivolumab plus ipilimumab has been approved to treat the unresectable MPM in treatment-naive patients as a first-line therapy by the FDA in October 2020. Immunotherapy is expected to be the best choice for MPM treatment. In the following article, the past treatment plan and the progress of immunotherapy for MPM will be reviewed.
\end{abstract}

Keywords: malignant pleural mesothelioma, immunotherapy, progression, ipilimumab, nivolumab

\section{Introduction}

In 2020 , the number of new cases of malignant mesothelioma globally is 30,870 , accounting for $0.2 \%$ of the world's new malignant tumors, and the number of deaths is 26,278 , accounting for $0.3 \%$ of the deaths of the global malignant tumors. ${ }^{1}$ Asbestos is an internationally recognized carcinogen. Wagner first reported the correlation between asbestos exposure and malignant pleural mesothelioma (MPM) in $1960 .^{2}$ Other studies suggest that peritoneal mesothelioma is rare upon non-occupational exposure, since the site is frequently exposed to asbestos. ${ }^{3}$ In some large-population countries, asbestos use has been approved since the late 1990s and is not apparently restricted. At present, a global catastrophe can be predicted for the next few decades, which may affect the global burden of mesothelioma. ${ }^{4}$ In recent years, the incidence rate of MPM has increased significantly worldwide, which is expected to reach the peak from 2015 to $2030 .^{5}$ In the United States, there are 2000 to 3000 new clinically diagnosed cases each year. ${ }^{6}$ The incidence rate even remains higher in Western Europe, with nearly 5000 new cases diagnosed per year. ${ }^{7}$

MPM is still a serious problem, with its difficult early diagnosis, clinical manifestations of diversity, high malignant degree and the fast progress, which is a kind of very difficult to treat diseases. The median survival time ranges as low as 8-14 months from diagnosis, and the 5 -year survival rate was less than $10 \%$, indicating a poor prognosis. ${ }^{8,9}$ Therefore, the treatment of MPM is still a difficult problem and a great challenge all over the world currently. With a particular emphasis placed on the immunotherapy, this article will mainly provide the latest advances in MPM therapy.

\section{Current Treatment Options for MPM}

Epithelioid variety is recognized as the most commonly seen MPM subtype by the World Health Organization (WHO) classification (2015) regarding pleural 
cancer, whereas sarcomatous and biphasic subtypes rank the second and third places, respectively. ${ }^{10}$ Surgical treatment is the preferred method for cancer, but most MPM patients can not receive surgical treatment when they are diagnosed. This is because that surgical treatment plays a debatable role in MPM, since radical surgery is suggested in the MARS study to be of questionable usefulness during trimodality therapy, which cannot achieve significant survival benefit and is associated with certain harmful effects. ${ }^{11}$ Although some studies have shown that patients with stage I-II sarcomatoid MPM can obtain longer overall survival (OS) from surgical treatment, the perioperative complications and mortality were significantly higher than those of non sarcomatoid MPM patients. ${ }^{12}$ Therefore, surgical treatment is not recommended. The results of a Phase III trial conducted by Vogelzang and others presented that patients receiving cisplatin combined with pemetrexed had longer survival significantly, with a median OS of 12.1 months, and at the same time the other group only receiving cisplatin is of 9.3 months. ${ }^{13}$ In February 2004, pemetrexed plus cisplatin was approved by the US FDA to be used to treat unresectable MPM as a first-line treatment. Vascular endothelial growth factor (VEGF) can promote new blood vessel formation, and it is also an autocrine growth factor for mesothelioma cells. The monoclonal antibody (mab)-mediated VEGF blockage suppresses mesothelioma cell proliferation. $^{14}$ In a randomized, controlled, and open label Phase III trial (IFCT-GFPC-0701 MAPS), the median OS was 18.8 months in group used pemetrexed, cisplatin, and bevacizumab contrasting 16.1 months in treatment group used pemetrexed combined with cisplatin. ${ }^{15}$ In general, adding bevacizumab to pemetrexed and cisplatin combined chemotherapy can improve the OS of MPM patients, and thus bevacizumab has been now recommended as a possible first-line treatment in combination with cisplatin and pemetrexed for unresectable MPM. ${ }^{16}$ As revealed by some Phase III clinical studies, pemetrexed plus carboplatin achieves superior survival benefit. $^{17,18}$ According to a study including altogether 1704 unresectable MPM cases, those receiving pemetrexed plus cisplatin had comparable OS and progression-free-survival (PFS) to those receiving pemetrexed plus carboplatin. ${ }^{18}$ A multicenter randomized controlled trial (MS01) has shown that vinorelbine monotherapy can also be used in platinum intolerant patients. ${ }^{19}$ A Phase III clinical trial shows that gemcitabine combining cisplatin can be used as an alternative therapy for patients who do not tolerate pemetrexed. ${ }^{20}$ For followup or later-line treatment, platinum stimulation test or single-agent used in combination with vinorelbine, gemcitabine and anthracyclines is frequently utilized, ${ }^{21}$ which does not achieve obvious survival benefits compared with the best supportive care. A retrospective study showed that gemcitabine and vinorelbine have certain benefits and can be used when there is no other option. $^{22}$ Currently, no registered second-line treatments are available, illustrating the urgent need for new treatment options.

\section{Immunotherapy}

Thanks to ICIs (Immune checkpoint inhibitors, ICIs) incorporation, great achievements have been attained in immunotherapy against different cancers. Immunotherapy aims to activate the immune system to trigger effective tumor-specific immune responses. At present, 3 kinds of ICIs can be used to suppress the immunosuppressive molecules below, including programmed death-1 (PD-1), PD-1 ligand-1 (PD-L1), and cytotoxic T-lymphocyte-associated protein 4 (CTLA-4). In a recent Phase III trial incorporating aCTLA-4 (ipilimumab) and aPD-1 (nivolumab) among the treatmentnaive MPM cases (namely, Checkmate-743), positive results were first obtained. On October $2^{\text {nd }}, 2020$, Opdivo (nivolumab) in combination with Yervoy (ipilimumab, Bristol-Myers Squibb Company) was approved by the US FDA to be used in the unresectable adult MPM patients as the first-line treatment. It is a real pleasure that this is the first drug approved by FDA for the systemic therapy of MPM in recent 16 years, and also the second kind of drug approved by FDA for systematic treatment of MPM in its history since the FDA approved pemetrexed combined with cisplatin in the treatment of MPM in 2004. Additionally, it has also experienced a long process of exploration to establish nivolumab combined with ipilimumab as the first-line treatment of MPM. A single-center Phase II study assessed the effectiveness and safety of nivolumab+ipilimumab in treating relapsed MPM patients. According to their findings, nivolumab+ipilimumab was markedly effective on treating relapsed MPM. 94\% cases were 
found to develop treatment-associated side effects, including $34 \% \quad(\mathrm{n}=35)$ with grade 3 treatmentassociated side effects. ${ }^{23}$ It is CheckMate 743 that really establishes "O $+\mathrm{Y}$ " (nivolizumab combined with ipilimumab) double immunotherapy combination for MPM. CheckMate 743 which is a multicentre, randomised, open-label and Phase III trial is the first and only Phase III clinical trial to demonstrate that first-line immunotherapy can improve the survival benefit of patients with MPM in comparison with chemotherapy. Compared with standard chemotherapy (pemetrexed plus cisplatin or carboplatin), navolizumab plus ipilimumab can significantly lower the risk of death in patients with unresectable MPM by $26 \%$. The result showed that compared with chemotherapy, nivolizumab combined with ipilimumab significantly improved OS, and the median OS was 18.1 months vs 14.1 months ( $\mathrm{HR}=0.74 ; 96.6 \% \mathrm{CI}: 0.60 \sim 0.91 ; \mathrm{P}=0.002)$. That is, OS was prolonged by 4 months compared with chemotherapy. The 1-year OS of the dual immunization regimen group was $68 \%$, and the 2 -year OS was $41 \%$. However, the 1-year OS of the chemotherapy regimen group was $58 \%$, and the 2 -year OS was only $27 \%$. It is really a leap forward that double immunization program enables over $40 \%$ of patients to obtain 2-year OS. Meanwhile, OS can always be beneficial regardless of the histological type of MPM patients. ${ }^{24,25}$ Based on the results of CheckMate-743, nivolumab combined with ipilimumab was approved by the FDA in October 2020 as the first-line treatment for adult patients with previously untreated, unresectable MPM. This regimen should be regarded as the new standard treatment for such patients no matter what histological subtype.

According to fundamental research on immunosuppressive tumor microenvironment (TME) of MPM, MPM was a possible favorable candidate for immunotherapy. ICIs are suggested to achieve certain promising effects. Other experimental studies show that immunotherapy has achieved certain results in MPM patients. DREAM was a multicentre, single-arm, openlabel, Phase II trial which is the first trial of PD-L1 inhibitor durvalumab combined with platinum-based chemotherapy in the first-line treatment of MPM. The study showed that $31(57 \% ; 95 \%$ CI 44-70) of 54 patients were alive and progression-free at 6 months and Durvalumab was continued for a maximum of 12 months. Compared with chemotherapy alone, chemotherapy combined with immunotherapy enhances the 6-month progression free survival rate and ORR (objective response rate), and the adverse reactions are tolerable. $^{26}$ The exploration of immunotherapy in MPM is first carried out in the second-line treatment. In the multi-center, open-label, non-comparative, randomized, Phase II trial IFCT-1501MAPS2, altogether 125 qualified cases were enrolled and divided into nivolumab ( $n=63)$ group and nivolumab plus ipilimumab $(n=62)$ group. According to their findings, the nivolumab plus ipilimumab group had the 1-year survival and median OS of $58 \%$ and 15.9 months (95\% CI: 10.7 months to unreached), respectively, while those in nivolumab alone group were $49.0 \%$ and 11.9 (95\% CI: $6.7-$ 17.7) months, respectively. ${ }^{27}$ The COFIRM study is the first Phase III clinical study of anti PD-1 monoclonal antibody and placebo control in patients with recurrent malignant mesothelioma ( $95 \%$ of MPM). According to the result, nivolumab treatment increases OS benefit and longer survival with nivolumab (events 232 [target 291]; median, 9.2 vs 6.6 months; HR, 0.72; 95\% CI, 0.55 $0.94 ; \mathrm{P}=0.02$ ). The 1 -year overall survival rate was $39.5 \%$ in the nivolumab group and $26.9 \%$ in the placebo group. PFS was 3.0 months in the nivolumab group and 1.8 months in the placebo group. The 1 -year progression free survival rates were $14.5 \%$ and $4.9 \%$, respectively, in the nivolumab group and the placebo group. ${ }^{28}$ According to the Phase II trial KEYNOTE-158, pembrolizumab exhibited persistent anticancer effect and tolerable toxic effect on advanced MPM cases, despite the PD-L1 status. $^{29}$ In the NivoMes Phase II study, enrolling 38 patients with relapsed MPM was treated with nivolumab, obtaining a 3-month DCR (Disease control rate, DCR) of $50 \%$ and an ORR of $24 \% .^{30}$ 3-Year Results of the MERIT Study showed that receiving nivolumab intravenously $240 \mathrm{mg}$ every 2 weeks was well tolerated and no new safety signals were found. Median OS and PFS were 17.3 and 5.9 months in thirtyfour patients enrolled who have been treated in one or two regimens with MPM before. The 2- and 3-year OS rates were $35.3 \%$ and $23.5 \% .^{31}$ At present, a Phase II, prospective, randomized controlled trial (RCT) is being carried out, which will help to evaluate the therapeutic effect of nivolumab plus standard chemotherapy on early MPM in the process of multimodal treatment. ${ }^{32}$ 
Table I The Studies on Immunotherapy of Malignant Pleural Mesothelioma

\begin{tabular}{|c|c|c|c|c|}
\hline $\begin{array}{l}\text { Study } \\
\text { Name }\end{array}$ & Treatment & Results & Safety & References \\
\hline $\begin{array}{l}\text { CheckMate } \\
74323\end{array}$ & $\begin{array}{l}\text { Nivolumab } \\
\text { +ipilimumab } \\
(n=303) v s \\
\text { chemotherapy } \\
\quad(n=302) .\end{array}$ & $\begin{array}{l}\text { Median OS: } 18.1 \text { vs } \mid 4 . \text { Imonths } \\
(\mathrm{HR}=0.74,96.6 \%, \mathrm{Cl} 0.60 \sim 0.91, \\
\mathrm{P}=0.0020) \\
2 \text {-year OS rate: } 41 \%(95 \% \mathrm{Cl} 35 \cdot \mathrm{I}- \\
46.5) \text { vs } 27 \%(21 \cdot 9-32 \cdot 4)\end{array}$ & $\begin{array}{l}\text { AEs (Grade 3-4):9I (30\%) of } 300 \text { vs } 9 \text { I (32\%) of } 284 . \\
\text { TRD:3 (I\%) vs I (<I\%) }\end{array}$ & {$[23,24]$} \\
\hline DREAM 25 & $\begin{array}{c}\text { Cisplatin } \\
+ \text { Durvalumab } \\
+ \text { pemetrexed }(n=54)\end{array}$ & $\begin{array}{l}\text { month } \mathrm{PFS}: 57 \%(3 \mathrm{I} \text { patients }) \\
\text { PR rate: } 48 \%(26 \text { patients })\end{array}$ & $\begin{array}{l}\text { AEs (Grade 3-4):neutropenia (7 [13\%] patients), } \\
\text { nausea (6 [1I\%]), and anaemia ( } 4[7 \%] \text { ) } \\
\text { Serious AEs: } 60 \text { in } 29 \text { participants (5possibly related } \\
\text { to durvalumab) }\end{array}$ & [25] \\
\hline $\begin{array}{l}\text { IFCT- } \\
\text { I50IMAPS2 }\end{array}$ & $\begin{array}{l}\text { Nivolumab }(n=63) \\
\text { vs } \\
\text { Nivolumab plus } \\
\text { ipilimumab }(n=62)\end{array}$ & $\begin{array}{l}\text { Median OS:II.9 (95\% Cl: } 6.7-17.7 \\
\text { months) vs I5.9(95\% Cl: } 10.7 \\
\text { months to not reached) months. } \\
\text { I-year OS rate: } 49 \% \text { vs } 58 \%\end{array}$ & $\begin{array}{l}\text { AEs (Grade } 3) \text { :asthenia (I }[2 \%] \text { vs } 3[5 \%]) \text {, } \\
\text { asymptomatic increase in aspartate aminotransferase } \\
\text { or alanine aminotransferase (0 vs } 4[7 \%]) \text {, and } \\
\text { asymptomatic lipase increase (2[3\%] vs I[2\%]). } \\
\text { TRD:0 vs } 3(5 \%)\end{array}$ & [26] \\
\hline $\begin{array}{l}\text { KEYNOTE- } \\
158\end{array}$ & Pembrolizumab & $\begin{array}{l}\text { Median OS: } 10 \cdot 0 \text { months }(95 \% \mathrm{Cl} \\
7 \cdot 6-13 \cdot 4) \\
\text { Median PFS:2 } 1 \text { months }(2 \cdot \mathrm{I}-3 \cdot 9) \text {. } \\
\text { OR:I4.3 months }(4.0 \text { to } 33.9+) \\
\text { I2-month ORR: } 60 \% \text {. }\end{array}$ & $\begin{array}{l}\text { AEs:82 (69\%) } \\
\text { AEs (Grade 3): } 19 \text { (16\%) } \\
\text { TRD:I(<1\%) }\end{array}$ & [28] \\
\hline MERIT & Nivolumab & $\begin{array}{l}\text { Median OS: } 17.3 \text { months. } \\
\text { PFS:5.9 months. } \\
\text { 2-year OS rates:35.3\% } \\
\text { 3-Year OS rate: } 23.5 \%\end{array}$ & No new safety signals were found & {$[31]$} \\
\hline NICITA & $\begin{array}{l}\text { Chemotherapy } \\
\text { combined with } \\
\text { nivolumab VS } \\
\text { Chemotherapy }\end{array}$ & Ongoing & Ongoing & [32] \\
\hline
\end{tabular}

Abbreviations: $\mathrm{AEs}$, adverse events; $\mathrm{Cl}$, confidence interval; OS, overall survival; ORR, overall response rate; PFS, progression-free survival; TRD, treatment-Related Death; DCR, disease control rate.

We have summarized and compared the results and safety of the above studies of immunotherapy (Table 1).

\section{Biomarkers for MPM}

The new era of MPM immunization has just arrived. The study of serum immune checkpoint and biomarker is conducive to judging the early prognosis and treatment of MPM. PD-1 shows expression in the activated $\mathrm{T}$ cells, which together with the PD-L1 and PDL2 ligands, regulates the functions of T-cell effectors. ${ }^{33}$ The PD-L1-positive MPM is markedly related to the dismal prognosis compared with PD-L1-negative MPM (median survival, $4.8-5.0$ vs $14.5-16.3$ months). ${ }^{34,35}$ As revealed by another article, aPD-1/aCTLA- 4 combined therapy profoundly enhanced $\mathrm{T}$ cell activation and growth, while these were not achieved in aPD-1 treatment alone. ${ }^{36}$ CheckMate 743 confirmed that patients with PD-L1 $\geq 1 \%(\mathrm{HR}=0.69)$ had more OS benefits and proved that the expression of PD-L1 may be a predictor of the efficacy of dual immunotherapy. ${ }^{23}$ Other studies have shown that some genes are also associated with PD-1 expression. Loss-of-function mutations in NF2 and LATS1/2 have been frequently observed, accounting for approximately $50 \%$ of MPM cases. ${ }^{37}$ Another study showed that Hippo-YAP activity is significantly correlated with PD-L1 expression in 
MPM samples, supporting anti-PD/PD-L1 immunotherapy for this subset of MPM patients. Furthermore, mutations affecting the core signaling molecules (LATS1/2, MST1) regulating Hippo-YAP activity in MPM patients presented better overall survival after ICB treatment. At the same time, they observed that in MPM with low NF2 expression, a high plasma B-cell infiltrative signature predicts better overall survival. ${ }^{38} \mathrm{~A}$ study on a unique phenotype of MPM immune microenvironment showed that p14/ARF-positive epithelioid mesotheliomas may mark a more aggressive pathological phenotype (higher nuclear grade and PD-L1 expression) and p14/ARF-negative tumors appear to have an immune microenvironment less sensitive to immune checkpoint inhibitors. $^{39}$

Genomic studies have shown that there is no specific driver gene mutation detected in MPM patients, and the inactivation of tumor suppressor genes is dominant, including CDKN2A/2B, BAP1, NF2, 1AST2 and other genes. Because it is more difficult to correct the inactivated tumor suppressor genes than to target the tumor driver genes, previous studies on targeted therapy of MPM have failed mostly. ${ }^{40}$ Gene BAP1 mutation is a common type of mutation in MPM patients. BAP1 gene mutation was detected in $45 \%-100 \%$ of MPM. BAP1 gene mutation mainly existed in epithelioid MPM, which was related to good prognosis. ${ }^{41}$ Gene BAP1 mutation leads to DNA double strand damage repair ability defect, resulting in genomic instability, which leads to the occurrence and development of diseases. ${ }^{42}$ Another retrospective article was conducted on 67 MPM cases, which discovered that BAP1 alterations occurred in the early stage of MPM occurrence and showed negative correlation with chemotherapy response through sequencing the samples acquired longitudinally. As confirmed in that article, BAP1 loss resulted in cisplatin resistance in vitro, which was mostly related to the decreased apoptosis. ${ }^{43}$ In vivo and in vitro experiments, the BAP1 gene mutation MPM cells were resistant to the tumor EZH2 (enhancer of zeste homolog 2) is sensitive. Tazemetostat, the first EZH2 inhibitor in the world, has been approved by FDA for metastatic or advanced epithelioid sarcoma that is not suitable for surgical resection. Currently, a Phase II clinical study in relapsed MPM shows that tazemetostat generates a good anti-tumor effect on patients with BAP1 gene mutation, and it is safe and reliable (NCT0280286). Meanwhile, other three pan HDAC inhibitors are being studied in MPM patients (NCT00365053, Nct00128102 and NCT00535951). ${ }^{44}$ In addition, there are also many biomarker-related experiments that have been proved to be related to the occurrence and development of MPM. A study using bioinformatics tools demonstrated that EZH2 and HMMR were found over-expressed in MPM tissues and the patients with elevated EZH2 and HMMR expressions had poor overall survival. ${ }^{41}$ Moreover, the study has confirmed that CDKN2A (p16) mutation is associated with poor prognosis. In sarcomatoid MPM, and the positive rate of CDKN2A (p16) almost remains $100 \%{ }^{45}$ There are also numerous ongoing and completed experimental studies on MPM biomarkers, ${ }^{46}$ aiming to better guide the clinical and bring the hope of cure to patients. We have summarized and compared the results and safety of the above studies of biomarkers associated with MPM (Table 2).

\section{Conclusion}

The diagnosis of many MPM cases is made at the advanced stage because of its aggressiveness and insidiousness. At present, molecular and clinical studies focus on prolonging patient survival and improving their life quality. Immunotherapy was first widely used in other cancers. After decades of trial failures, immunotherapy is rapidly emerging as an important tool for the treatment of MPM. Typically, the Checkmate-743 study has brought favorable news for MPM cases. In October 2020, the FDA approved Nivolumab plus ipilimumab for treating treatment-naive unresectable MPM cases as a first-line treatment. Recently, the immunotherapy has altered the treatment and further enhanced the survival of these patients. Immune checkpoint inhibitors are a new research direction in the treatment of MPM. Meanwhile, the research of molecular markers also provides a new idea for the treatment of MPM. In the future, the treatment of MPM still needs to be studied. Gene detection and targeted therapy may also be one of the treatment options for MPM in the future. We hope that this article gives useful information to physicians in the area. 
Table 2 Conclusions of Biomarkers Associated with MPM

\begin{tabular}{|c|c|l|c|}
\hline Biomarkers & \multicolumn{1}{|c|}{ Studies } & \multicolumn{1}{c|}{ Results } & References \\
\hline PD-LI & $\begin{array}{c}\text { Mansfield A S et al./ } \\
\text { Cedrés S et al. }\end{array}$ & $\begin{array}{l}\text { MPM with PD-LI positivity has significantly been associated with a poorer prognosis than } \\
\text { PD-LI negative MPM }\end{array}$ & [34,35] \\
\hline $\begin{array}{c}\text { CTLA-4 } \\
\text { +PD-I }\end{array}$ & $\begin{array}{c}\text { Joanne M. } \\
\text { Mankor et al. }\end{array}$ & $\begin{array}{l}\text { APD-I/aCTLA-4 combination treatment induced a profound increase in proliferation and } \\
\text { activationof T cells, which was not observed upon aPD-I monotherapy. }\end{array}$ & [36] \\
\hline BAPI & $\begin{array}{c}\text { Betti M et al } \\
\text { /Oehl K et al. }\end{array}$ & $\begin{array}{l}\text { I. BAPI gene mutation was detected in 45\% - I00\% of MPM. } \\
\text { 2. Loss of BAPI leads to resistance to cisplatinin vitroand. }\end{array}$ & [4I,43] \\
\hline PI4/ARF & $\begin{array}{c}\text { Pezzuto F } \\
\text { I. PI4/ARF-positive epithelioid mesotheliomas may mark a more aggressive pathological } \\
\text { phenotype (higher nuclear grade and PD-LI expression) } \\
\text { 2. PI4/ARF-negative tumors seem to have an immune microenvironment less sensitive to } \\
\text { immune checkpoint inhibitors36. }\end{array}$ & [39] \\
\hline $\begin{array}{c}\text { NF2 and } \\
\text { Hippo-YAP }\end{array}$ & $\begin{array}{c}\text { Hmeljak J et al./ } \\
\text { Haitang }\end{array}$ & $\begin{array}{l}\text { I. Loss-of-function mutations in NF2 and LATSI/2 account for approximately 50\% of MPM } \\
\text { cases. } \\
\text { 2. Hippo-YAP activity is significantly correlated with PD-LI expression. } \\
\text { 3. Mutations affecting the core signaling molecules (LATSI/2, MSTI) regulating Hippo-YAP } \\
\text { activity in MPM patients show better overall survival after ICB treatment. } \\
\text { 4. Low NF2 expression, a high plasma B-cell infiltrative signature predicts better overall } \\
\text { survival. }\end{array}$ & [37,38] \\
\hline
\end{tabular}

\section{Data Sharing Statement}

Not applicable.

\section{Consent for Publication}

Not applicable.

\section{Acknowledgment}

The authors would like to thank the National Natural Science Foundation of China (No. 81403220 and No.81904151), Tianjin Health and family planning-high level talent selection and training project, Tianjin Science and Technology Plan Projects (No. 17ZXMFSY00190) and Tianjin Traditional Chinese Medicine Research Project, Tianjin health and family planning commission (No. 2017003).

\section{Author Contributions}

All authors read and approved the final manuscript. All authors made substantial contributions to conception and design, acquisition of data, or analysis and interpretation of data; took part in drafting the article or revising it critically for important intellectual content; agreed to submit to the current journal; gave final approval of the version to be published; and agree to be accountable for all aspects of the work.

\section{Funding}

This work is supported by the National Natural Science Foundation of China (No. 81403220 and No.81904151),
Tianjin Health and family planning-high level talent selection and training project, Tianjin Science and Technology Plan Projects (No. 17ZXMFSY00190) and Tianjin Traditional Chinese Medicine Research Project, Tianjin health and family planning commission (No. 2017003).

\section{Disclosure}

The authors report no conflicts of interest.

\section{References}

1. Sung H, Ferlay J, Siegel RL, et al. Global cancer statistics 2020: GLOBOCAN estimates of incidence and mortality worldwide for 36 cancers in 185 countries. CA Cancer J Clin. 2021;71 (3):209-249.

2. Wagner JC, Sleggs CA, Marchand P. Diffuse pleural mesothelioma and asbestos exposure in the North Western Cape Province. $\mathrm{Br}$ J Ind Med. 1960;17:260-271. doi:10.1136/oem.17.4.260

3. Orriols R, Tarrés J, Albertí-Casas C, et al. Malignant asbestos-related disease in a population exposed to asbestos. Am J Ind Med. 2020;63 (9):796-802.

4. Chimed-Ochir O, Arachi D, Driscoll T, Lin RT, Takala J, Takahashi K. Burden of mesothelioma deaths by national income category: current status and future implications. Int $J$ Environ Res Public Health. 2020;17. doi:10.3390/ijerph17186900.

5. Robinson BM. Malignant pleural mesothelioma: an epidemiological perspective. Ann Cardiothorac Surg. 2012;1(4):491-496. doi:10.3978/ j.issn.2225-319X.2012.11.04

6. Kindler HL, Ismaila N, Armato SG 3rd, et al. Treatment of malignant pleural mesothelioma: American Society of Clinical Oncology clinical practice guideline. $J$ Clin Oncol. 2018;36(13):1343-1373. doi:10.1200/JCO.2017.76.6394 
7. Rusch VW, Giroux D, Kennedy C, et al. Initial analysis of the international association for the study of lung cancer mesothelioma database. J Thorac Oncol. 2012;7(11):1631-1639. doi:10.1097/ JTO.0b013e31826915f1

8. Scherpereel A, Astoul P, Baas P, et al. Guidelines of the European Respiratory Society and the European Society of Thoracic Surgeons for the management of malignant pleural mesothelioma. Eur Respir J. 2010;35(3):479-495. doi:10.1183/09031936.00063109

9. Beckett P, Edwards J, Fennell D, Hubbard R, Woolhouse I, Peake MD. Demographics, management and survival of patients with malignant pleural mesothelioma in the National Lung Cancer Audit in England and Wales. Lung Cancer. 2015;88(3):344-348. doi:10.1016/j.lungcan.2015.03.005

10. Galateau-Salle F, Churg A, Roggli V, et al. The 2015 World Health Organization classification of tumors of the pleura: advances since the 2004 classification. J Thoracic Oncol. 2016;11(2):142-154.

11. Treasure T, Lang-Lazdunski L, Waller D, et al. Extra-pleural pneumonectomy versus no extra-pleural pneumonectomy for patients with malignant pleural mesothelioma: clinical outcomes of the Mesothelioma and Radical Surgery (MARS) randomised feasibility study. Lancet Oncol. 2011;12(8):763-772. doi:10.1016/S14702045(11)70149-8

12. Kim S, Bull DA, Garland L, et al. Is there a role for cancer-directed surgery in early-stage sarcomatoid or biphasic mesothelioma? Ann Thorac Surg. 2019;107(1):194-201.

13. Vogelzang NJ, Rusthoven JJ, Symanowski J, et al. Phase III study of pemetrexed in combination with cisplatin versus cisplatin alone in patients with malignant pleural mesothelioma. J Clin Oncol. 2003;21 (14):2636-2644. doi:10.1200/JCO.2003.11.136

14. Remon J, Reguart N, Corral J, Lianes P. Malignant pleural mesothelioma: new hope in the horizon with novel therapeutic strategies. Cancer Treat Rev. 2015;41(1):27-34. doi:10.1016/j. ctrv.2014.10.007

15. Zalcman G, Mazieres J, Margery J, et al. Bevacizumab for newly diagnosed pleural mesothelioma in the Mesothelioma Avastin Cisplatin Pemetrexed Study (MAPS): a randomised, controlled, open-label, phase 3 trial. Lancet. 2016;387(10026):1405-1414. doi:10.1016/S0140-6736(15)01238-6

16. Levin PA, Dowell JE. Spotlight on bevacizumab and its potential in the treatment of malignant pleural mesothelioma: the evidence to date. Onco Targets Ther. 2017;10:2057-2066. doi:10.2147/OTT. S113598

17. Katirtzoglou N, Gkiozos I, Makrilia N, et al. Carboplatin plus pemetrexed as first-line treatment of patients with malignant pleural mesothelioma: a phase II study. Clin Lung Cancer. 2010;11(1):30-35.

18. Santoro A, O'Brien ME, Stahel RA, et al. Pemetrexed plus cisplatin or pemetrexed plus carboplatin for chemonaive patients with malignant pleural mesothelioma: results of the International Expanded Access Program. J Thoracic Oncol. 2008;3(7):756-763.

19. Van Haarst JMW, Baas P, Manegold C, et al. Multicentre phase II study of gemcitabine and cisplatin in malignant pleural mesothelioma. Br J Cancer. 2002;86(3):342-345.

20. Arrieta O, López-Macías D, Mendoza-García VO, et al. A phase II trial of prolonged, continuous infusion of low-dose gemcitabine plus cisplatin in patients with advanced malignant pleural mesothelioma. Cancer Chemother Pharmacol. 2014;73(5):975-982.

21. Sinn K, Mosleh B, Hoda MA. Malignant pleural mesothelioma: recent developments. Curr Opin Oncol. 2021;33(1):80-86.

22. Abdel-Rahman O, Kelany M. Systemic therapy options for malignant pleural mesothelioma beyond first-line therapy: a systematic review. Expert Rev Respir Med. 2015;9(5):533-549.

23. Disselhorst MJ, Quispel-Janssen J, Lalezari F, et al. Ipilimumab and nivolumab in the treatment of recurrent malignant pleural mesothelioma (INITIATE): results of a prospective, single-arm, phase 2 trial. Lancet Respir Med. 2019;7(3):260-270.
24. Baas P, Scherpereel A, Nowak AK, et al. First-line nivolumab plus ipilimumab in unresectable malignant pleural mesothelioma (CheckMate 743): a multicentre, randomised, open-label, phase 3 trial. The Lancet. 2021;397(10272):375-386.

25. Uprety D. CheckMate 743: a glimmer of hope for malignant pleural mesothelioma. Clin Lung Cancer. 2021;22(2):71-73.

26. Nowak AK, Lesterhuis WJ, Kok PS, et al. Durvalumab with first-line chemotherapy in previously untreated malignant pleural mesothelioma (DREAM): a multicentre, single-arm, phase 2 trial with a safety run-in. Lancet Oncol. 2020;21(9):1213-1223.

27. Scherpereel A, Mazieres J, Greillier L, et al. Nivolumab or nivolumab plus ipilimumab in patients with relapsed malignant pleural mesothelioma (IFCT-1501 MAPS2): a multicentre, open-label, randomised, non-comparative, phase 2 trial. Lancet Oncol. 2019;20(2):239-253.

28. Fennell D, Ottensmeier C, Califano R, et al. PS01. 11 nivolumab versus placebo in relapsed malignant mesothelioma: the CONFIRM Phase 3 trial. J Thoracic Oncol. 2021;16(3):S62.

29. Yap TA, Nakagawa K, Fujimoto N, et al. Efficacy and safety of pembrolizumab in patients with advanced mesothelioma in the open-label, single-arm, phase 2 KEYNOTE-158 study. Lancet Respir Med. 2021.

30. Quispel-Janssen J, Noort V, Vries J, et al. PD-1 blockade with nivolumab in patients with recurrent Malignant Pleural Mesothelioma. J Thoracic Oncol. 2018;13:10.

31. Fujimoto N, Okada M, Kijima T, et al. Clinical efficacy and safety of nivolumab in Japanese patients with malignant pleural mesothelioma: 3-year results of the MERIT study. JTO Clin Res Rep. 2021;2(3):100135.

32. Shah R, Klotz LV, Chung I, et al. A phase II trial of nivolumab with chemotherapy followed by maintenance nivolumab in patients with pleural mesothelioma after surgery: the NICITA study protocol. Clin Lung Cancer. 2021;22(2):142-146.

33. Pardoll DM. The blockade of immune checkpoints in cancer immunotherapy. Nat Rev Cancer. 2012;12(4):252-264.

34. Mansfield AS, Roden AC, Peikert T, et al. B7-H1 expression in malignant pleural mesothelioma is associated with sarcomatoid histology and poor prognosis. J Thoracic Oncol. 2014;9(7):1036-1040.

35. Cedrés S, Ponce-Aix S, Zugazagoitia J, et al. Analysis of expression of programmed cell death 1 ligand 1 (PD-L1) in malignant pleural mesothelioma (MPM). PLoS One. 2015;10(3):e0121071.

36. Mankor JM, Disselhorst MJ, Poncin M, et al. Efficacy of nivolumab and ipilimumab in patients with malignant pleural mesothelioma is related to a subtype of effector memory cytotoxic T cells: translational evidence from two clinical trials. EBioMedicine. 2020;62:103040.

37. Hmeljak J, Sanchez-Vega F, Hoadley KA, et al. Integrative molecular characterization of malignant pleural mesothelioma. Cancer Discov. 2018;8(12):1548-1565.

38. Yang H, Hall SRR, Sun B, et al. NF2 and Canonical Hippo-YAP pathway define distinct tumor subsets characterized by different immune deficiency and treatment implications in human pleural mesothelioma. Cancers. 2021;13(7):1561.

39. Pezzuto F, Lunardi F, Vedovelli L, et al. P14/ARF-positive malignant pleural mesothelioma: a phenotype with distinct immune microenvironment. Front Oncol. 2021;11.

40. Mutti L, Peikert T, Robinson BWS, et al. Scientific advances and new frontiers in mesothelioma therapeutics. J Thoracic Oncol. 2018;13 (9):1269-1283.

41. Betti M, Aspesi A, Ferrante D, et al. Sensitivity to asbestos is increased in patients with mesothelioma and pathogenic germline variants in BAP1 or other DNA repair genes. Genes Chromosomes Cancer. 2018;57(11):573-583.

42. Yang H, Xu D, Schmid RA, et al. Biomarker-guided targeted and immunotherapies in malignant pleural mesothelioma. Ther Adv Med Oncol. 2020;12:1758835920971421.

43. Oehl K, Vrugt B, Wagner U, et al. Alterations in BAP1 are associated with cisplatin resistance through inhibition of apoptosis in malignant pleural mesothelioma. Clin Cancer Res. 2021;27(8):2277-2291. 
44. Liu X, Qian K, Lu G, et al. Identification of genes and pathways involved in malignant pleural mesothelioma using bioinformatics methods. BMC Med Genomics. 2021;14(1):1-11.

45. Wu D, Hiroshima K, Matsumoto S, et al. Diagnostic usefulness of p16/CDKN2A FISH in distinguishing between sarcomatoid mesothelioma and fibrous pleuritis. Am J Clin Pathol. 2013;139 (1):39-46.
46. Cakiroglu E, Senturk S. Genomics and functional genomics of malignant pleural mesothelioma. Int J Mol Sci. 2020;21(17):6342.

\section{Publish your work in this journal}

OncoTargets and Therapy is an international, peer-reviewed, open access journal focusing on the pathological basis of all cancers, potential targets for therapy and treatment protocols employed to improve the management of cancer patients. The journal also focuses on the impact of management programs and new therapeutic

Submit your manuscript here: https://www.dovepress.com/oncotargets-and-therapy-journal agents and protocols on patient perspectives such as quality of life, adherence and satisfaction. The manuscript management system is completely online and includes a very quick and fair peer-review system, which is all easy to use. Visit http://www.dovepress.com/ testimonials.php to read real quotes from published authors. 